\title{
The enlightenments from ITMIG Consensus on WHO histological classification of thymoma and thymic carcinoma: refined definitions, histological criteria, and reporting
}

\author{
Jie Wu', Wentao Fang ${ }^{2}$, Gang Chen ${ }^{1}$ \\ ${ }^{1}$ Department of Pathology, Zhongshan Hospital, Fudan University, Shanghai 200032, China; ${ }^{2}$ Department of Thoracic Surgery, Shanghai Chest \\ Hospital, School of Medicine, Shanghai Jiao Tong University, Shanghai 200030, China \\ Contributions: (I) Conception and design: G Chen, W Fang; (II) Administrative support: W Fang; (III) Provision of study materials or patients: All \\ authors; (IV) Collection and assembly of data: J Wu; (V) Data analysis and interpretation: All authors; (VI) Manuscript writing: All authors; (VII) \\ Final approval of manuscript: All authors. \\ Correspondence to: Gang Chen. Department of Pathology, Zhongshan Hospital, Fudan University, 180 Fenglin Road, Shanghai 200032, China. \\ Email: chestpathology@126.com.
}

\begin{abstract}
The World Health Organization (WHO) histological classification of the thymoma and thymic carcinoma (TC) has been criticized for poor interobserver reproducibility or inconsistencies in the routine pathological diagnosis. The International Thymic Malignancy Interest Group (ITMIG) panel achieved an agreement to maintain the widely accepted WHO framework but to refine historic definitions and histological criteria, and further introduce some new terms with the aim to improve interobserver reproducibility. This review addresses the enlightenments we can get from the ITMIG consensus on the WHO histological classification of the thymoma and TC, which may be helpful for most pathologists.
\end{abstract}

Keywords: Thymic epithelial tumor (TET); histology; reporting

Submitted Oct 05, 2015. Accepted for publication Nov 10, 2016.

doi: $10.21037 /$ jtd.2016.01.84

View this article at: http://dx.doi.org/10.21037/jtd.2016.01.84

\section{Introduction}

The World Health Organization (WHO) classification is the most widely used histological classification of thymomas and thymic carcinomas (TCs). However, the WHO classification has been criticized for poor interobserver reproducibility or inconsistencies in the routine diagnosis (1-3), when encountering some certain cases: (I) thymomas with features intermediate between prototypic subtypes (borderland cases); (II) tumors with atypia, high mitotic activity, and necrosis; (III) tumors showing more than one histological pattern. To address these issues at an interdisciplinary conference organized by the International Thymic Malignancy Interest Group (ITMIG) in New York, in March 2011, the participants including 18 pathologists, two surgeons, and one oncologist reviewed prototypic and difficult-to-classify thymic epithelial tumors (TETs) and achieved the consensus to refine histological criteria for better management. The article about the consensus statement was published on Fournal of Thoracic Oncology, in May 2014 (4).

The ITMIG panel achieved an agreement to maintain the widely accepted WHO framework but to improve historic definitions and introduce some new terms with the aim to improve interobserver reproducibility:

(I) The WHO classification has been criticized for imprecise descriptions of $\mathrm{A}$ and $\mathrm{AB}$ thymoma and for calling them benign (5-7). At the consensus workshop there was agreement that $\mathrm{A}$ and $\mathrm{AB}$ thymomas are tumors of low malignant potential. The data of Chinese Alliance for Research in Thymomas (ChART), 1,930 cases of TETs from 10 hospitals from 1994 to 2012, showed that 10-year overall survival of type $A$ and $A B$ thymomas (accounted for $4.4 \%$ and $22.8 \%$ ), were $92.4 \%$ 


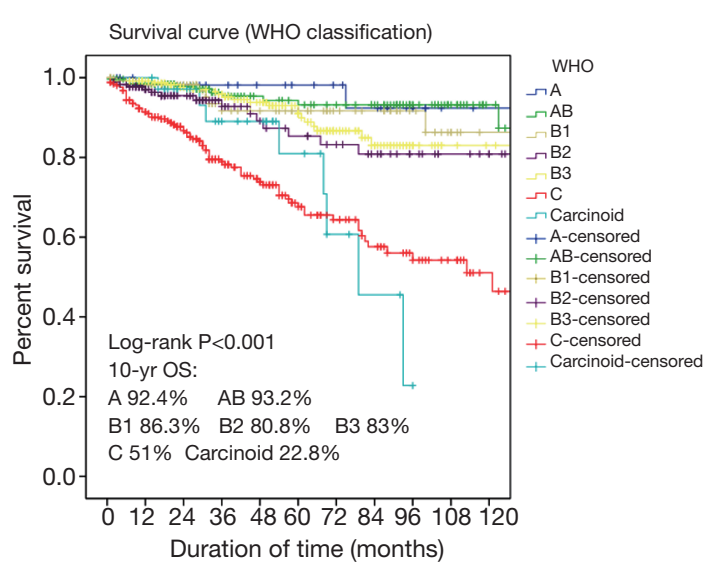

Figure 1 The 10-year overall survival of 1,930 cases of TETs from 10 hospitals from 1994 to 2012.

and $93.2 \%$ respectively (Figure 1 );

(II) Taking into account that thymomas with heterogeneous histological features composed of different subtypes are very common, there was consensus that the term "combined thymoma" should be abandoned. Instead, the diagnosis in such tumors should follow an approach analogous to Gleason scoring listing all subtypes starting with the predominant component; minor components should be reported with $10 \%$ increments. Of note, $\mathrm{AB}$ thymoma is a distinct entity for which the $10 \%$ rule does not apply. For scientific and statistical purposes, thymoma components of $0 \%$ to $10 \%$ can be neglected, and the given tumor classified according to the dominant component. If thymic tumors comprising a carcinoma component should be different from the reporting of thymomas: such tumors should in the first place be labeled as carcinomas with listing of the proportion, differentiation, and grade, followed by the list of the thymoma components;

(III) In the WHO classification the imprecise definition of $\mathrm{AB}$ thymomas was "organotypic thymic epithelial neoplasms composed of a mixture of lymphocyte-poor type A thymoma component and a more lymphocyterich type B-like component analogous to B1 or B2 thymomas" (8). Now the potentially confusing term "B-like area" is replaced by "lymphocyte-rich" component in $\mathrm{AB}$ thymomas, and the criticized statement given in the WHO classification that lymphocyte-rich areas in $\mathrm{AB}$ thymomas harbor polygonal tumor cells is replaced: tumor cells in such areas are typically spindly or oval;

(IV) The new concept of atypical type A thymoma was posed in the consensus statement. Agreed criteria of "atypia" were increased mitotic activity (4 or more per 10 high power field) and "true" (coagulative) tumor necrosis (in contrast to ischemic or biopsy-induced necrosis). Other criteria, such as hypercellularity, enlarged hyperchromatic nuclei, large nucleoli, increased Ki67 index, and extent of atypical areas, were difficult to quantify or could not be agreed upon. Actually some of type A thymomas indeed showed overt invasiveness and metastasis $(5,6)$, there was agreement that the type A thymoma family includes a small subset of aggressive tumors. Nevertheless, further subdivision of type A thymoma into different entities in analogy to the B1, B2, and B3 paradigm appears to premature before reliable data available (9).

The panel members agreed on the description of major (indispensable) and minor (typical) diagnostic criteria by tables, instead of the "narrative style" of the WHO classification. As supplement, "galleries of figures" illustrated different-to-classify tumors at the "borderlands" between prototypic cases. On account of the interest in borderland cases with differential diagnostic value, 72 cases were selected for review at the consensus workshop, only 58 could finally be fully evaluated due to time restrictions. The differential diagnosis on these borderland cases mainly focused on type $\mathrm{A}$ and $\mathrm{AB}$ thymoma, type $\mathrm{B} 1$ and $\mathrm{B} 2$ thymoma, type B3 thymoma and TC.

\section{Differential diagnosis on type A thymoma}

\section{Distinguishing type A thymoma from AB thymoma}

In the WHO classification the description of type A thymoma was that there was no or only few $\mathrm{T}$ cells with expression of CD3 and CD5. Immature $\mathrm{T}$ cells with expression of CD1a and CD99 could also present in type A thymoma. In the consensus statement the panels agreed to quantify the proportion of immature $\mathrm{T}$ cells of type A thymoma. It should harbor no or only few $\mathrm{TdT}+\mathrm{T}$ cells (easy to count) (grade 1) or a moderate amount of $\mathrm{Td} T+\mathrm{T}$ cells (I could count if I had to) (grade 2) in 10\% or less of a given biopsy (Table 1). Moderate numbers of $\mathrm{TdT}+\mathrm{T}$ cells above the arbitrary $10 \%$ threshold in available biopsies or any area with abundant (impossible to count) $\mathrm{Td} T+\mathrm{T}$ cells (grade 3 ) would favor a diagnosis of $\mathrm{AB}$ thymoma over type 
Table 1 Major and criteria of "conventional" type A thymomas

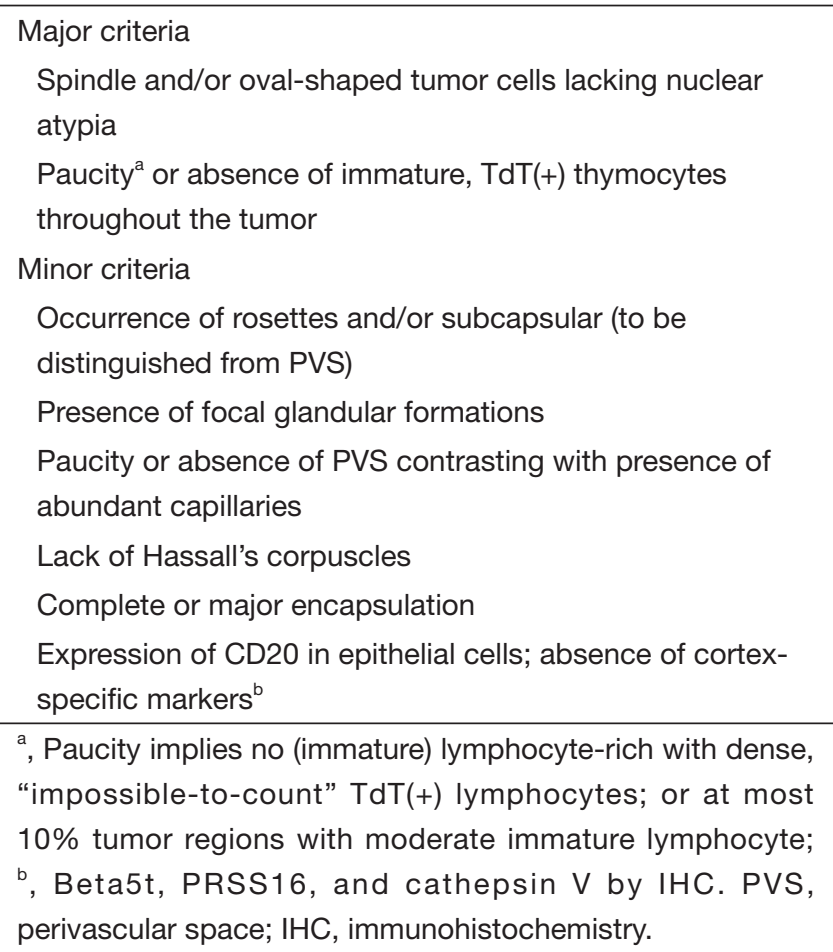

A thymoma. The role of immunohistochemistry (IHC) was emphasized in the consensus statement: epithelial cells of $\mathrm{AB}$ thymomas express both cortical and medullary markers in an intermingled pattern, whereas type A thymomas lack cortical markers (Table 2) (10).

\section{Distinguishing type A thymoma from spindle cell B3 thymoma}

In the WHO description Th reticulin fibers was applied for differential diagnosis on type A thymoma and spindle cell B3 thymoma (8). In type A thymoma reticulin fiber often presented around single tumor cell with expression of Laminin and collagen IV, whereas B3 thymoma lack of reticulin fibers. The consensus statement proposed reticulin fiber did not reliably distinguish type A from spindle B3 thymomas, while the difference on morphology was more valuable. Prominent and abundant perivascular spaces (PVSs) would strongly favor a diagnosis of type B3 thymoma, whereas uniform nuclei, abundance of capillary vessels, rosette formation, cystic spaces, and epithelial expression of CD20 would favor type A thymoma. Nevertheless, distinction between atypical type A thymoma and spindle cell B3 thymoma can be more difficult because nuclear atypia is present in both, and immunohistochemical studies may be further required.

\section{Differential diagnosis on type B thymomas}

\section{Distinguishing B1 thymomas from B2 thymomas}

B1 thymomas closely mimic normal thymus (NT) at both low and high magnification, with presence of prominent "medullary islands" that contain epithelial cells with or without Hassall's corpuscles; a majority of mature, TdT (-) T cells; and scattered CD20+ mature B cells. Medullary islands can also occur in B2 thymoma. PVS and abundant $\mathrm{TdT}+\mathrm{T}$ cells occur in both B1 and B2 thymomas, but PVSs are often inconspicuous in B1 thymomas. The distinguishing features of B2 thymomas are: (I) increased number of epithelial cells compared with NT often visible at low magnification; and (II) epithelial cell clusters (defined as at least three contiguous epithelial cells). On immunostaining, the network of epithelial cells in B2 thymoma is significantly denser. In the WHO description there was significant difference on tumor cell size, shape and nucleolus between $\mathrm{B}$ thymomas, while the consensus achieved is that nuclear size and atypia of epithelial cells are not helpful and reliable distinguishing features.

\section{Distinguishing B2 thymoma from B3 thymoma}

According to the statistical results from ChART, the prognosis of B2 thymoma was worse than B3 (10-year overall survival: $80.8 \%$ vs. $83.0 \%$ ) (Figure 1). As a "rule of thumb" H\&E-stained B2 and B3 thymomas give a "blue" vs. "pink" impression, respectively, due to the prominent $\mathrm{T}$ cells in B2 versus B3 thymomas. In the WHO classification previously described distinguishing criteria such as nuclear size and PVS are not helpful for this distinction.

\section{Differential diagnosis between thymoma and TC}

\section{Distinguishing B3 thymoma from thymic squamous cell carcinoma (TSCC)}

In general, TCs show the same histological features as analogous extra-TCs (Table 3) (11-14). B3 thymomas typically show lobular growth, conspicuous PVS, minor/ moderate nuclear atypia, lack of intercellular bridges, presence of $\mathrm{Td} \mathrm{T}+$ immature $\mathrm{T}$ cells, and lack of expression of CD5, CD117, GLUT1, and MUC1 in neoplastic epithelial cells (15-18). Nevertheless, some following 
Table 2 Major and minor histological features encountered in type A and AB thymomas

\begin{tabular}{|c|c|c|}
\hline Features & Type A thymoma & Type AB thymoma \\
\hline \multicolumn{3}{|l|}{ Major criteria } \\
\hline $\begin{array}{l}\text { Biphasic pattern at low magnification due to variable lymphocyte } \\
\text { content }\end{array}$ & No & Common $^{a}$ \\
\hline High epithelial cell content & Yes & Yes \\
\hline Spindle or oval epithelial cells ${ }^{b}$ & Yes & Yes \\
\hline \multicolumn{3}{|l|}{ Minor criteria } \\
\hline Small lobular growth pattern & No & Rare \\
\hline Large lobular growth pattern & Common & Common \\
\hline Perivascular spaces & Rarely present & Rarely present \\
\hline
\end{tabular}

a , these feature are minor criteria in type AB thymoma; ${ }^{b}$, atypia in type AB thymoma has not been addressed so far; ${ }^{c}$, as defined in Table 1; ${ }^{d}$, detection of medullary islands is usually clear-out on hematoxylin-eosin staining but may require IHC, particularly when Hassall's corpuscles are missing; ${ }^{e}$, in lymphocyte-rich areas, usually with lack of Hassall's corpuscles; ${ }^{f}$, Beta5t, PRSS16, and cathepsin V (detectable by IHC in epithelial cells within lymphocyte-rich areas). IHC, immunohistochemistry.

Table 3 Criteria for the histological diagnosis of TC

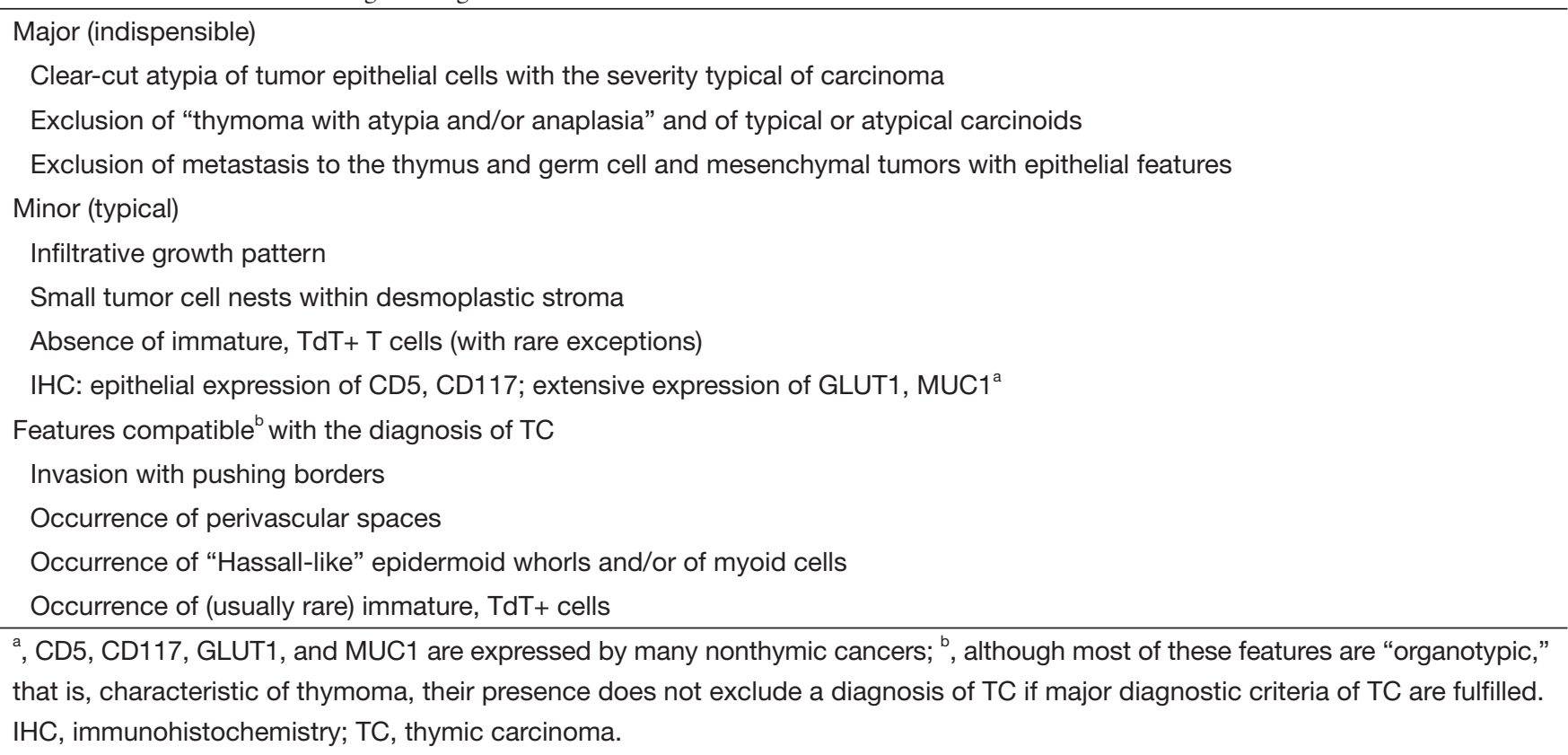

equivocal situations still needed clarification. If tumors that lack $\mathrm{TdT}+\mathrm{T}$ cells in the available histological material but otherwise show features of typical B3 thymomas and CD5/ CD117 negativity should be called B3 thymomas. Despite the expression of CD5, CD117, MUC1, or GLUT1 in an otherwise typical B3 thymoma, we should not change the diagnosis to TC (Table 4). If tumors with absence of two features of thymic squamous cell carcinoma (TSCC) (clear- 
Table 4 Major and minor histological feature of type B1 versus B2 thymomas

\begin{tabular}{lll}
\hline Features & Type B1 thymoma & Type B2 thymoma \\
\hline Major criteria & & Rarely present \\
Thymus-like pattern throughout & Consistently present & Occasionally present $^{\mathrm{a}}$ \\
Medullary islands (+/- Hassal's corpuscles) & Consistently present & Yes \\
Confluence of epithelial cells in cortical areas ${ }^{\mathrm{b}}$ & No (like in the NT) & Yes \\
Absence of type A areas (even if $<10 \%)$ & Yes & Common \\
Minor criteria & & Rare \\
Small lobular growth pattern & Rare & Commonly present \\
Large lobular growth pattern & Common & Commonly present \\
Perivascular spaces & Yes & Denser than in NT \\
Keratin ${ }^{c}$ network like in NT
\end{tabular}

${ }^{a}$, These features are, therefore, minor criteria of type B2 thymomas; ${ }^{b}$, defined as at least three contiguous epithelial cells; ${ }^{c}$, on immunostaining. NT, normal thymus.

cut nuclear atypia and intercellular bridges) and lack of an important feature of $\mathrm{B} 3$ thymomas ( $\mathrm{Td} T+\mathrm{T}$ cells), they were tentatively labeled as "B3/TSCC borderline TETs".

\section{Distinguishing atypical type $A$ thymoma from spindle cell TC}

As to this borderland, the panel members thought there were no efficient approaches to differential diagnosis. Analysis of TdT is not helpful, as absence of TdT+ thymocytes does not exclude a diagnosis of atypical type A thymoma. Morphologically classical type A thymomas should not be reclassified as TC only on the basis of CD117 and CD5 expression. New "subtype-specific" markers are needed to study this unresolved borderland. The statistical data of ChART revealed that TC patients had lowest prognosis, with $51 \%$ 10-year overall survival. As a new subtype, whether the prognosis of atypical A thymoma is worse or not, need more data to verify that (Figure 1).

\section{Conclusions}

The consensus achieved by the panel of ITMIG on refined definitions and histological criteria is helpful for interobserver reproducibility. The borderland cases often occurred in spectrum of type $\mathrm{A}$ and $\mathrm{AB}$ thymoma, type $\mathrm{B}$ thymoma, and TC. The tables that list major and minor diagnosis criteria and the galleries of figures that illustrate different-to-classify TETs make pathologists easy to grasp and practice on diagnosis. The proposal of new concepts of atypical type A thymoma and B3/TSCC borderline
TETs further supplement the WHO classification. IHC play an important role in differential diagnosis, especially on thymomas and TCs, while as an auxiliary approach, the panelists still emphasize the morphology features, including nuclear atypia, mitotic activity, and tumor necrosis, when encountering the borderland cases.

\section{Acknowledgements}

Funding: This study was supported by the Shanghai Science and Technique Committee (134119a3200).

\section{Footnote}

Conflicts of Interest: The authors have no conflicts of interest to declare.

\section{References}

1. Suster S, Moran CA. Problem areas and inconsistencies in the WHO classification of thymoma. Semin Diagn Pathol 2005;22:188-97.

2. Rieker RJ, Hoegel J, Morresi-Hauf A, et al. Histologic classification of thymic epithelial tumors: comparison of established classification schemes. Int J Cancer 2002;98:900-6.

3. Verghese ET, den Bakker MA, Campbell A, et al. Interobserver variation in the classification of thymic tumours--a multicentre study using the WHO classification system. Histopathology 2008;53:218-23.

4. Marx A, Ströbel P, Badve SS, et al. ITMIG consensus 
statement on the use of the WHO histological classification of thymoma and thymic carcinoma: refined definitions, histological criteria, and reporting. J Thorac Oncol 2014;9:596-611.

5. Jain RK, Mehta RJ, Henley JD, et al. WHO types A and $\mathrm{AB}$ thymomas: not always benign. Mod Pathol 2010;23:1641-9.

6. Moran CA, Kalhor N, Suster S. Invasive spindle cell thymomas (WHO Type A): a clinicopathologic correlation of 41 cases. Am J Clin Pathol 2010;134:793-8.

7. Wick MR. Histopathologic prognosis of thymomas: another example of medical surrogacy. Am J Clin Pathol 2010;134:703-5.

8. Müller-Hermelink HK, Engel P, Kuo TT, et al. Tumoursofthethymus. In: Travis WD, Brambilla E, Müller-Hermelink HK, eds. World Health Organization Classification of Tumours: Pathology and Genetics. Tumours of the lung, pleura, thymus and heart. Lyon: IARC Press, 2004.

9. Nonaka D, Rosai J. Is there a spectrum of cytologic atypia in type a thymomas analogous to that seen in type B thymomas? A pilot study of 13 cases. Am J Surg Pathol 2012;36:889-94.

10. Ströbel P, Hartmann E, Rosenwald A, et al. Corticomedullary differentiation and maturational arrest in thymomas. Histopathology 2014;64:557-66.

11. Ströbel P, Hohenberger P, Marx A. Thymoma and thymic carcinoma: molecular pathology and targeted therapy. J Thorac Oncol 2010;5:S286-90.

12. Marx A, Rieker R, Toker A, et al. Thymic carcinoma: is it a separate entity? From molecular to clinical evidence. Thorac Surg Clin 2011;21:25-31. v-vi.

13. Weissferdt A, Moran CA. Thymic carcinoma, part 1: a clinicopathologic and immunohistochemical study of 65 cases. Am J Clin Pathol 2012;138:103-14.

14. Moran CA, Suster S. Thymic carcinoma: current concepts and histologic features. Hematol Oncol Clin North Am 2008;22:393-407.

15. Kojika M, Ishii G, Yoshida J, et al. Immunohistochemical differential diagnosis between thymic carcinoma and type B3 thymoma: diagnostic utility of hypoxic marker, GLUT-1, in thymic epithelial neoplasms. Mod Pathol 2009;22:1341-50.

16. Kaira K, Endo M, Abe M, et al. Biologic correlation of 2-[18F]-fluoro-2-deoxy-D-glucose uptake on positron emission tomography in thymic epithelial tumors. J Clin Oncol 2010;28:3746-53.

17. Hishima T, Fukayama M, Fujisawa M, et al. CD5 expression in thymic carcinoma. Am J Pathol 1994;145:268-75.

18. Henley JD, Cummings OW, Loehrer PJ Sr. Tyrosine kinase receptor expression in thymomas. J Cancer Res Clin Oncol 2004;130:222-4.
Cite this article as: Wu J, Fang W, Chen G. The enlightenments from ITMIG Consensus on WHO histological classification of thymoma and thymic carcinoma: refined definitions, histological criteria, and reporting. J Thorac Dis 2016;8(4):738743. doi: $10.21037 /$ jtd.2016.01.84 